\title{
Modelo para avaliação do desempenho da segurança viária através da simulação microscópica
}

\author{
Flávio José Craveiro Cunto; Frank F. Saccomanno²
}

\begin{abstract}
Resumo: O uso da microssimulação em estudos de segurança viária tem sido investigado mais frequentemente nas últimas duas décadas. Em tese, essa ferramenta pode atuar como plataforma para o desenvolvimento de uma abordagem mais mecanística dos eventos que precedem a ocorrência de acidentes de trânsito. Este artigo apresenta um modelo para avaliação do desempenho da segurança viária através da microssimulação. O modelo utiliza o aplicativo VISSIM $^{\odot}$ versão 4.3 como plataforma de simulação e estima interações longitudinais e transversais entre veículos ao longo do tempo, a partir do índice de potencial para acidentes (CPI). A utilidade do modelo proposto foi ilustrada através de sua aplicação em interseções isoladas semaforizadas ou não. Os resultados indicam que a introdução do semáforo aumentou a frequência e severidade das interações longitudinais e, reduziu o número de veículos interagindo transversalmente. Estes resultados confirmam o potencial considerável para o uso da microssimulação em estudos de segurança viária.
\end{abstract}

\begin{abstract}
The use of microsimulation in safety studies has been more frequently investigated over the last two decades. In theory, this tool can serve as platform for the development of a more mechanistic approach regarding the events preceding a crash. This paper presents a model for assessing the road safety performance using microsimulation. The model applies the software VISSIMC 4.3 as simulation platform and estimates rear-end and angled interactions for different vehicle over time via the crash potential index (CPI). The usefulness of the proposed model has been illustrated throughout its application to signalized and unsignalized isolated intersections. The results indicate that the signalization increased both frequency and severity for rear-end interactions, decreasing, on the other hand, the number of angled interactions. These results also confirm the potential for using microscopic simulation in road safety studies.
\end{abstract}

\section{INTRODUÇÃO}

A simulação microscópica tem sido utilizada nas últimas três décadas como ferramenta de análise do desempenho operacional de sistemas de transporte com relativo sucesso. Mais recentemente, o seu potencial para aplicações em análises do desempenho da segurança viária passou a ser investigado de maneira sistemática (Gettman e Head, 2003; Archer, 2005; Huguenin et al., 2005). Uma das características deste tipo de abordagem é o fato de ser possível a investigação de diferentes estratégias em um ambiente virtual evitando assim, distúrbios no tráfego existente ou custos de implantação desnecessários. Diferentemente dos estudos de segurança que aplicam modelos estatísticos de previsão de acidentes, a microssimulação representa em sua essência uma abordagem pró-ativa. Desta forma, não se faz necessária a ocorrência de certo número de acidentes para a detecção de problemas de segurança viária (Barceló et al., 2003; Darzentas et al., 1980).

Os estudos de segurança viária utilizando a microssimulação baseiam-se em indicadores proxy de segurança que procuram representar de maneira contínua o

\footnotetext{
${ }^{1}$ Flávio José Craveiro Cunto, Departamento de Engenharia de Transportes, Universidade Federal do Ceará, Fortaleza, CE, Brasil. (e-mail: flaviocunto@det.ufc.br).

${ }^{2}$ Frank F. Saccomanno, Department of Civil and Environmental Engineering, University of Waterloo, Waterloo, Canadá. (e-mail: saccoman@uwaterloo.ca).
}

Manuscrito recebido em 2/6/2010 e aprovado para publicação em 8/7/2010. Este artigo é parte de TRANSPORTES, volume XVIII, número 3, setembro de 2010. ISSN: 2237-1346 (online). nível de interação espaço-temporal entre veículos (Gettman e Head, 2003; Archer, 2005; Huguenin et al., 2005). Este tipo de abordagem traz na sua essência um paradigma similar ao encontrado nos estudos de análise de conflitos de tráfego, ou seja, o desempenho de segurança viária é materializado através de uma série de eventos temporais que variam de passagens sem perturbação pelo tráfego à ocorrêcia de acidentes de trânsito, conhecido como o continuum de segurança viária (Hydén, 1987; Hauer, 2002). Os principais problemas para consolidar essa abordagem, até certo ponto mais racional, estão diretamente relacionados à falta de objetividade dos indicadores proxy, necessidade de informações detalhadas para a obtenção dos mesmos e necessidade de validação do indicador proposto com situações comprovadas de elevado risco de colisão (Hauer, 1978; Archer, 2005; Saunier e Sayed, 2007).

Em tese, a microssimulação pode atuar como plataforma para a obtenção e validação de indicadores proxy que utilizem informações veiculares detalhadas ao longo do tempo e do espaço. Vale ressaltar, entretanto, que os resultados obtidos precisam ser analisados em um contexto mais abrangente que considere os conceitos de conflitos/interações de tráfego, simplificações dos algoritmos de simulação e a validade relativa destes indicadores para refletir a segurança viária.

O objetivo deste artigo é apresentar um modelo para a estimação do desempenho da segurança de redes de tráfego tendo como base a simulação microscópica. Para tanto, faz-se inicialmente uma revisão dos principais estudos de segurança viária que utilizaram me- 
didas proxy de segurança obtidas através da microssimulação. Em seguida, apresenta-se a configuração geral do modelo proposto e seus principais componentes. Finalmente, o uso do modelo é ilustrado através de sua aplicação em interseções isoladas.

\section{USO DA MICROSSIMULAÇÃO EM ESTUDOS DE SEGURANÇA VIÁRIA}

O nível da segurança associado aos diversos componentes dos sistemas de transporte é frequentemente medido em termos do número de acidentes de trânsito observados ao longo de um certo intervalo de tempo. Problemas relacionados à qualidade dos dados disponíveis, além de questões metodológicas associadas à natureza extremamente infrequente e randômica deste tipo de evento, têm incentivado o desenvolvimento de pesquisas de indicadores alternativos de segurança viária (indicadores proxy). Estes indicadores podem culminar com uma abordagem mais mecanística dos eventos que precedem a ocorrência de acidentes de trânsito.

Pesquisas recentes sugerem que indicadores proxy obtidos continuamente ao longo do trajeto dos veículos podem fornecer medidas mais objetivas e abrangentes do desempenho da segurança viária (Minderhoud e Bovy, 2001; Huguenin et al., 2005). Uma das desvantagens destes indicadores é a dificuldade de obtenção de informações detalhadas dos veículos envolvidos de forma contínua. Desta forma, a microssimulação tem sido investigada como plataforma potencial para a obtenção de dados representativos que permitam o cálculo dos diversos indicadores proxy para diversos cenários de tráfego (Cunto e Loureiro, 2009).

Cooper e Ferguson (1976) e Darzentas et al. (1980) foram pioneiros na tentativa de criação de modelos de simulação de conflitos de tráfego em interseções. Apesar de simplificado, em virtude das limitações computacionais da época, o modelo permitiu concluir que a severidade dos conflitos medida em termos da taxa de desaceleração para evitar a colisão, aumentou com a média e o desvio padrão da velocidade dos veículos.

Mais recentemente, estudos sistemáticos utilizando pacotes comerciais de simulação como o PARAMICS, VISSIM, AIMSUN, dentre outros, são encontrados com certa frequência na literatura (Gettman e Head, 2003; Archer, 2005; Huguenin et al.; 2005; Xin et al., 2008). De maneira geral, os esforços de pesquisa convergem para quatro questões fundamentais:

- Algoritmos tradicionais de car-following, aceitação de brecha e mudança de faixa não foram desenvolvidos especificamente para a modelagem dos diversos eventos que podem ocasionar colisões e outras situações de risco. Quais são os algoritmos existentes mais adequados para esse tipo de modelagem? É possível o desenvolvimento de algoritmos mais complexos em termos de comportamento humano ao interagir com outros veículos?

- Os indicadores proxy devem reflitir com fidelidade as variáveis circunstanciais e a sequência de eventos que podem gerar acidentes de trânsito. Quais são os indicadores proxy de segurança viária mais adequados para o uso neste tipo de aplicação?

- O sucesso dessa abordagem depende naturalmente da possibilidade de calibração e validação dos algoritmos em termos do indicador proxy de segurança utilizado. É possível a calibração e validação de indicadores proxy de segurança viária?

- Apesar da relação aparente entre os indicadores proxy e acidentes de trânsito, o sucesso do modelo depende de um estudo mais conclusivo a respeito dessa relação. É possível o desenvolvimento de uma metodologia que venha a validar os indicadores proxy de segurança simulados com eventos "reais" relativos à ocorrência de situações de risco como conflitos de tráfego e acidentes?

As questões apresentadas motivaram a criação de um modelo para a avaliação do desempenho da segurança viária utilizando a microssimulação. As características gerais do modelo são apresentas a seguir.

\section{MODELO MICROSCÓPICO DE AVALIAÇÃO DA SEGURANÇA VIÁRIA}

A premissa básica do modelo proposto é a de que o desempenho de segurança viária pode ser considerado como função da geometria da via, atributos do tráfego e condições ambientais, interagindo com diferentes usuários ao longo do tempo. Essa interação gera um nível de "turbulência" que pode ser associado ao desempenho de segurança viária seguindo, desta forma, a noção de continuum de segurança proposta por Hydén (1987). O esquema conceitual do modelo é apresentado na Figura 1.

No modelo da Figura 1, a plataforma de simulação atua na representação dos componentes de entrada no processo de condução, através de seus submodelos de representação da rede (geometria), entrada de dados de fluxo e controle do tráfego (condições de tráfego) e modelos estocásticos para representar o comportamento dos condutores. O processo de condução é considerado a partir dos algoritmos de car-following, aceitação de brechas e mudanças de faixa normalmente presentes nos microssimuladores. As alterações nos fatores ambientais não são consideradas diretamente nos 


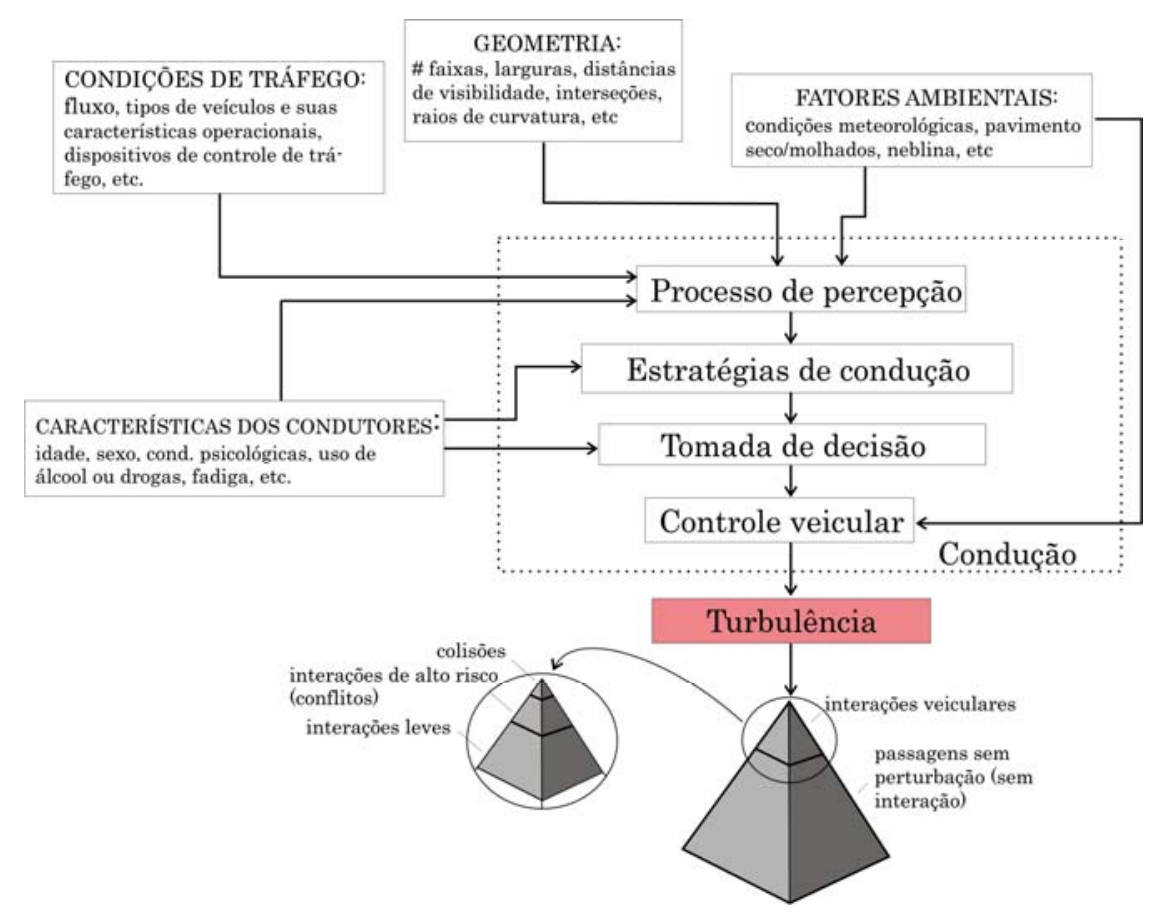

Figura 1. Esquema conceitual do modelo de avaliação do desempenho da segurança viária

algoritmos dos microssimuladores. Em consequência disso, diferenças comportamentais por variações nesses aspectos devem ser obtidas através de uma calibração dos parâmetros dos algoritmos.

No âmbito do modelo, a turbulência no tráfego gerada durante o processo é estimada a partir da ocorrência de eventos marcados pela existência de rota de colisão entre dois veículos, chamados de interações veículares. O veículo que inicia a interação seja desacelerando para realizar uma conversão ou parar diante de um semáforo, mudando de faixa ou aceitando uma brecha para cruzar uma interseção, é chamado de veículo-estímulo (VE). Uma vez iniciada a manobra o condutor diretamente afetado pelo VE pode reagir para evitar uma possível colisão, sendo chamado de veículo-reação (VR). Nesse contexto, as interações veiculares podem ser separadas em interações longitudinais (veículos na mesma direção) e transversais. Os algoritmos de car-following e mudança de faixa são utilizados nas interações longitudinais, enquanto que os algoritmos de aceitação de brecha são fundamentais para a representação das interações transversais.

A frequência e a severidade das interações são medidas utilizando o indicador proxy proposto por Cunto (2008) denominado índice de pontencial para acidentes (CPI-crash potential index). O CPI é calculado a partir da taxa de desaceleração necessária para evitar a colisão (DRAC-deceleration rate to avoid the crash), que por sua vez, é determinada para o VR a cada intervalo de tempo em que os veículos estão interagindo. A DRAC é calculada pela diferença de velocidade e espaçamento entre os veículos aplicando as equações de movimento da mecânica newtoniana. As expressões de DRAC para interações longitudinais e transversais podem ser encontradas em Cunto (2008).

O CPI considera ainda um componente estocástico que procura representar a capacidade máxima de desaceleração (MADR - maximum available deceleration rate) de um veículo em função de características como massa e sistema de freios, assim como de condições ambientais como tempo bom, chuvoso, presença de neve, etc. O CPI é obtido usando a seguinte expressão:

$$
C P I_{i}=\frac{\sum_{t=t_{i}}^{t f_{i}} P\left(M A D R^{\left(a_{1}, a_{2}, \ldots, a_{n}\right)} \leq D R A C_{i, t}\right) \cdot \Delta t \cdot b}{T_{i}}
$$

em que,

$C P I_{i}$ : índice de potencial para acidentes (crash potential índex) para o veículo $i$;

$M A D R_{i}$ : taxa máxima de desaceleração disponível para o veículo $i$

$D R A C_{i, t}$ : taxa de desaceleração para evitar a colisão para o veículo $i$ no instante $t$;

$t i_{i}$ : intervalo de tempo inicial para o veículo $i$;

$t_{f}$ : intervalo de tempo final para o veículo $i$;

$a_{1}, a_{2}, \ldots, a_{n}$ : variáveis representando o tipo de veículo, condições ambientais, etc.;

$\Delta t$ : intervalo de tempo de observação [s];

$T_{i}$ : tempo total observado para o veículo $i[\mathrm{~s}]$;

$b$ : variável de estado $(0=$ sem interação, $1=$ veículos interagindo).

Partindo da Equação 1, observa-se que cada veículo terá seu nível de turbulência medido pelo somatório dos intervalos de tempo $(\Delta \mathrm{t})$ expostos a um determinado nível de interação, dividido pelo tempo total do veículo na simulação. Através dos conceitos de DRAC e MADR, pode-se ainda definir interações se- 
veras ou conflitos de tráfego quando em um determinado instante da simulação ocorrer $\mathrm{P}(\mathrm{MADR}<\mathrm{DRAC})$ $>0,5$. De maneira mais prática, ao iniciar a simulação, cada veículo terá seu próprio MADR gerado a partir de uma distribuição probabilística em função do tipo de veículo e das características ambientais e, sempre que DRAC $>$ MADR, um conflito será associado àquele veículo.

O modelo então proposto é formado pela plataforma de simulação responsável pela modelagem do processo de condução dos veículos na rede e por outro subsistema que estima a turbulência gerada pelo processo de interação dos veículos. A estrutura geral do modelo é ilustrada na Figura 2.

Conforme ressaltado anteriormente, a plataforma de simulação é um elemento fundamental no esquema proposto no sentido de que seus subsistemas e algoritmos devem reproduzir, com boa fidelidade, o processo de condução de veículos em uma corrente de tráfego. Atualmente, o grupo de algoritmos mais adequados a este tipo de aplicação inclui aqueles baseados em estudos sobre a capacidade de percepção humana à aproximação de objetos, denominados modelos psico-físicos ou de ponto de ação.

O aplicativo comercial de microssimulação VISSIMC (2007) utiliza um dos modelos psicofísicos que foi desenvolvido por Wiedmman na década de 70 e aperfeiçoado em 1992 (Wiedemman, 1974; Wiedemann e Rieter, 1992). O modelo considera 4 estágios de condução percebidos pelos condutores: 1) condução livre; 2) aproximação; 3) perseguição; e 4) freio de emergência. A transição entre os estágios ocorre através de 6 limites da percepção humana a saber: 1) distância desejada entre veículos parados
(AX); espaçamento mínimo desejado para pequenas diferenças de velocidade (ABX); limite de percepção de diferença de velocidade para grandes espaçamentos (SDV); limite de percepção de distanciamento durante o processo de perseguição (SDX); limite de percepção de pequena diferença de velocidade durante o processo de aproximação (CLDV); e limite de percepção de pequena diferença de velocidade durante o processo de distanciamento (OPDV).

Além das vantagens inerentes aos seus algoritmos de modelagem, o VISSIM $^{\circledR}$ foi escolhido como plataforma de simulação por uma série de características consideradas importantes para o modelo de avaliação de desempenho proposto, dentre elas:

- Arquivos de saída configuráveis;

- Permitir pequenos intervalos de simulação $(0,1 \mathrm{~s})$;

- Permitir a geração de veículos de várias dimensões, além de considerar diferentes características operacionais entre veículos de categorias distintas e entre veículos da mesma categoria;

- Alta flexibilidade na definição do fluxo e composição do tráfego, incluindo movimentos de conversão e fluxos com variação temporal;

- Permitir a modelagem de reduções de velocidade para movimentos de conversão;

- Possibilitar o acesso de informações e alterações em parâmetros durante a simulação através de uma interface de programação do aplicativo (API);

- Apresentar um módulo de controle de tráfego com alto nível de detalhamento, incluindo semáforos isolados, coordenados e atuados pelo tráfego.

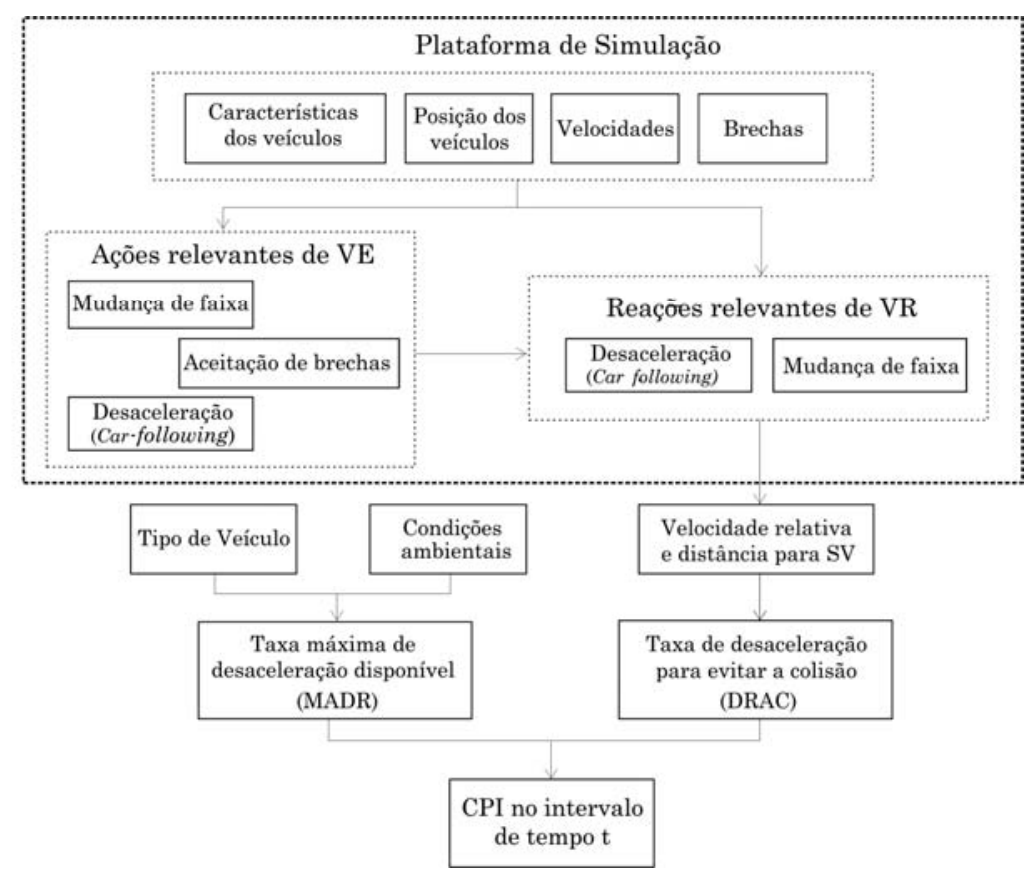

Figura 2. Modelo de avaliação do desempenho da segurança viária 
Tabela 1. Características de fluxo veicular consideradas

\begin{tabular}{|c|c|c|c|c|c|c|c|c|c|}
\hline \multirow[b]{2}{*}{ Nível $^{(*)}$} & \multicolumn{4}{|c|}{ Volume (vph) } & \multicolumn{2}{|c|}{ Placa PARE } & \multicolumn{3}{|c|}{ Semaforizada } \\
\hline & Leste & Oeste & Norte & Sul & $\begin{array}{c}\text { Principal } \\
\operatorname{LOS}^{(* *)}\end{array}$ & $\begin{array}{l}\text { Secundária } \\
\text { LOS }\end{array}$ & $\begin{array}{c}\text { Ciclo } \\
\text { (s) }\end{array}$ & $\begin{array}{c}\text { Principal } \\
\text { LOS }\end{array}$ & $\begin{array}{c}\text { Secundária } \\
\text { LOS }\end{array}$ \\
\hline 1 & 300 & 300 & 100 & 100 & $\mathrm{~A}$ & $\mathrm{C}$ & 40 & $\mathrm{~A}$ & $\mathrm{~A}$ \\
\hline 2 & 400 & 400 & 100 & 100 & A & $\mathrm{C}$ & 40 & A & A \\
\hline 3 & 500 & 500 & 100 & 100 & A & D & 40 & A & A \\
\hline 4 & 600 & 600 & 100 & 100 & A & $\mathrm{F}$ & 40 & A & A \\
\hline 5 & 700 & 700 & 100 & 100 & A & $\mathrm{F}$ & 40 & A & A \\
\hline 6 & 800 & 800 & 100 & 100 & A & $\mathrm{F}$ & 40 & $\mathrm{~B}$ & A \\
\hline 7 & 900 & 900 & 100 & 100 & A & $\mathrm{F}$ & 45 & $\mathrm{~B}$ & A \\
\hline 8 & 1000 & 1000 & 100 & 100 & B & $\mathrm{F}$ & 45 & B & A \\
\hline 9 & 1100 & 1100 & 100 & 100 & B & $\mathrm{F}$ & 50 & B & $\mathrm{B}$ \\
\hline 10 & 1200 & 1200 & 100 & 100 & B & $\mathrm{F}$ & 60 & B & B \\
\hline
\end{tabular}

A qualidade das análises realizadas com os dados produzidos no ambiente da microssimulação depende diretamente da habilidade da plataforma em reproduzir medidas de desempenho similares às obtidas a partir de dados de campo (calibração e validação). No âmbito do modelo proposto, a calibração/validação da plataforma deve privilegiar medidas de desempenho que reflitam a natureza do indicador proxy de segurança. A formulação matemática do indicador proxy CPI utiliza uma combinação do espaçamento e diferença de velocidade entre dois veículos ao longo do tempo e, além disso, esse índice pode ser especificado de forma agregada (CPI médio) ou desagregada, como no caso de veículos em conflito (DRAC $>$ MADR). Em consequência disso, a calibração/validação da plataforma a partir de medidas de desempenho tradicionais (tempo de viagem, fluxo médio, velocidade média, etc.) pode não ser válida em estudos de segurança viária.

O indicador CPI foi calibrado/validado por Cunto (2008) com o VISSIM $^{\odot} 4.3$ atuando como plataforma de simulação. O CPI médio e o número de veículos em conflito foram utilizados como medidas de desempenho em um procedimento hierárquico que utilizou análise fatorial e algoritmo genético para produzir uma expressão linear relacionando os parâmetros dos modelos com o CPI médio. A metodologia foi aplicada com relativo sucesso em uma via arterial com 4 semáforos atuados e coordenados e em um trecho de 640m de extensão da rodovia HWY101, ambos na Califórnia. Apesar dos resultados promissores, o procedimento utilizou dados bastante desagregados normalmente não disponíveis e, em virtude da área bastante reduzida, não se sabe ao certo se o mesmo procedimento pode ser aplicado em redes maiores.

Não obstante a relação intuitiva entre os indicadores proxy e acidentes de trânsito, uma investigação formal dessa relação pode confirmar a hipótese de que o risco de colisões aumenta diretamente com o nível de turbulência do sistema (indicadores proxy). Considera-se portanto, a validação do indicador proxy simulado utilizando situações "reais" de risco como conflitos ou acidentes de trânsito, como uma etapa adicional necessária ao sucesso do modelo proposto.

Para o indicador CPI, Cunto (2008) apresenta três testes que objetivaram comprovar a ligação entre o indicador CPI e acidentes de trânsito. Nos dois primeiros testes, para uma amostra de 27 acidentes, as mesmas condições de fluxo e velocidade para um período de cinco minutos antes da ocorrência foram reproduzidas individualmente no ambiente da simulação. $\mathrm{O}$ valor do CPI foi comparado ao longo dos cinco minutos que antecederam a colisão (teste 1) e também, comparado com o CPI obtido para o mesmo local em condições médias de fluxo e velocidade em um dia "normal" para os mesmos cinco minutos (teste 2). O último teste procurou estabelecer uma relação matemática mais agregada entre a taxa de acidentes anual em períodos de 1 hora e o CPI obtido através da simulação do mesmo intervalo de tempo (teste 3). Os resultados mostraram que: o CPI aumentou a medida que o momento da colisão se aproximou (teste 1); o CPI para as condições de colisão foi maior do que o CPI estimado para as condições médias de um dia "normal" (teste 2); e o CPI horário pôde ser diretamente relacionado à taxa anual de acidentes por veic.km do segmento de rodovia testado.

\section{APLICAÇÃO DO MODELO EM INTERSEÇÕES ISOLADAS}

As interseções viárias são locais caracterizados pela existência de correntes de tráfego convergindo para um ponto comum. Estes locais demandam um alto grau de interação entre o usuário e seu veículo, a via e outros usuários, criando um ambiente favorável à o- 
corrência de acidentes de trânsito. Dentre as questões mais controversas nesse cenário estão: Que tipo de controle minimiza a ocorrência de acidentes? Quais os benefícios em termos de segurança comparados ao custo de implantação do controle?

$\mathrm{Na}$ tentativa de contribuir para a solução das questões acima e, com o objetivo secundário de ilustrar a aplicação do modelo proposto, o mesmo foi aplicado para investigar os impactos na segurança viária ocasionados pela introdução da sinalização semafórica em interseções isoladas previamente controladas por placas PARE. Além da mudança no tipo de sinalização, procurou-se também investigar a sensibilidade do modelo com relação às mudanças no fluxo veicular.

A interseção codificada consistiu de quatro aproximações em ângulo reto com duas faixas de tráfego por sentido (3,5m por faixa) na via principal e 1 faixa por sentido na via secundária. A área total considerada foi de $100 \mathrm{~m}$ a montante e a jusante da interseção em cada direção. O plano de simulação foi projetado considerando 10 níveis diferentes de volume para cada aproximação e os planos semafóricos foram estimados considerando a semi-atuação nas vias secundárias através do software Synchro 7. Foi ainda assumida uma taxa de conversões à esquerda e à direita de $10 \%$ do fluxo de cada aproximação sendo $5 \%$ para cada direção. $\mathrm{Na}$ interseção semaforizada foram permitidas conversões à esquerda sem estágio direcional específico (esquerda forçada). A Tabela 1 apresenta um re- sumo dos parâmetros de tráfego usados na aplicação.

$\mathrm{O}$ desempenho da segurança foi modelado com o aplicativo $\operatorname{VISSIM}^{\odot} 4.3$, utilizando parâmetros de entrada calibrados e validados para o índice de potencial para acidentes (CPI) apresentados no trabalho de Cunto (2008). Para cada nível de volume, 15 simulações foram executadas, cada uma com duração de 20 minutos, sendo os 5 primeiros minutos descartados (warmup time). Pela configuração completa dos movimentos da interseção, um total de 32 áreas de possíveis interações transversais foram detectadas. Vale ressaltar que as interações longitudinais podem ocorrer em qualquer ponto ao longo da área da interseção.

O nível de turbulência em cada simulação foi medido em termos de quatro indicadores, a saber: CPI por veículo (CPI/veic); $85^{\circ}$ percentil do CPI (CPI85); porcentagem de veículos interagindo (CPI $>0)$; e porcentagem de veículos em conflito (DRAC $>$ MADR). A distribuição assumida para MADR foi normal truncada com média e desvio padrão de $12,7 \mathrm{~m} / \mathrm{s}^{2}$ e $4.2 \mathrm{~m} / \mathrm{s}^{2}$, respectivamente. Os valores de cada indicador, calculados a partir da média das 15 simulações executadas, estão apresentados na Tabela 2. A seguir, uma série de considerações será feita partindo da análise dos resultados apresentados nessa tabela.

Nas interações longitudinais (possíveis colisões traseiras), a introdução do semáforo resultou em aumento no $\mathrm{CPI} /$ veic, CPI85 e porcentagem de veículos interagindo em todos os níveis de volume testado. Esse

Tabela 2. Indicadores de desempenho da segurança obtidos para interseções semaforizadas e não semaforizadas

\begin{tabular}{|c|c|c|c|c|c|c|c|c|c|c|}
\hline & \multirow[b]{2}{*}{ Nível } & \multirow{2}{*}{$\begin{array}{c}\text { Veículos } \\
\text { simulados }\end{array}$} & \multicolumn{4}{|c|}{ Interações longitudinais } & \multicolumn{4}{|c|}{ Interações transversais } \\
\hline & & & CPI/veh & CPI85 & $\begin{array}{c}\text { \%Veic. } \\
\text { interagindo }\end{array}$ & $\begin{array}{c}\text { \%Veic. } \\
\text { em conflito }\end{array}$ & CPI/veh & CPI85 & $\begin{array}{c}\text { \%Veic. } \\
\text { interagindo }\end{array}$ & $\begin{array}{c}\text { \%Veic. } \\
\text { em conflito }\end{array}$ \\
\hline \multirow{9}{*}{ 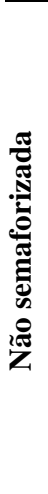 } & 1 & 198 & $1,27 \mathrm{E}-08$ & $1,48 \mathrm{E}-08$ & 19,8 & 0,00 & $1,45 \mathrm{E}-05$ & $9,98 \mathrm{E}-09$ & 4,8 & 0,03 \\
\hline & 2 & 247 & $5,87 \mathrm{E}-09$ & $6,60 \mathrm{E}-09$ & 22,4 & 0,00 & $1,98 \mathrm{E}-05$ & 2,63E-08 & 4,5 & 0,00 \\
\hline & 3 & 300 & $1,17 \mathrm{E}-08$ & 9,80E-09 & 27,7 & 0,00 & $8,77 \mathrm{E}-05$ & $2,36 \mathrm{E}-08$ & 6,2 & 0,02 \\
\hline & 4 & 347 & $1,85 \mathrm{E}-06$ & $7,44 \mathrm{E}-09$ & 31,6 & 0,02 & $1,36 \mathrm{E}-05$ & $1,42 \mathrm{E}-08$ & 6,7 & 0,02 \\
\hline & 5 & 396 & $7,48 \mathrm{E}-08$ & $5,12 \mathrm{E}-09$ & 37,1 & 0,00 & $3,14 \mathrm{E}-08$ & $1,83 \mathrm{E}-08$ & 6,9 & 0,00 \\
\hline & 6 & 454 & $9,90 \mathrm{E}-06$ & $7,04 \mathrm{E}-09$ & 42,7 & 0,02 & $6,30 \mathrm{E}-05$ & $2,72 \mathrm{E}-08$ & 7,1 & 0,06 \\
\hline & 7 & 506 & $2,58 \mathrm{E}-08$ & 4,51E-09 & 47,2 & 0,00 & $8,53 \mathrm{E}-05$ & $3,16 \mathrm{E}-08$ & 7,0 & 0,04 \\
\hline & 8 & 555 & $2,64 \mathrm{E}-06$ & 4,24E-09 & 51,1 & 0,02 & $1,82 \mathrm{E}-05$ & $3,00 \mathrm{E}-08$ & 5,9 & 0,02 \\
\hline & 9 & 610 & $1,88 \mathrm{E}-06$ & $3,06 \mathrm{E}-09$ & 56,5 & 0,01 & 1,02E-04 & $3,03 \mathrm{E}-08$ & 5,1 & 0,06 \\
\hline \multirow{10}{*}{ 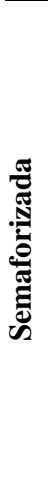 } & 1 & 199 & $4,08 \mathrm{E}-08$ & $6,35 \mathrm{E}-08$ & 27,2 & 0,00 & 1,59E-09 & $1,59 \mathrm{E}-09$ & 0,2 & 0,00 \\
\hline & 2 & 250 & $6,22 \mathrm{E}-08$ & $5,67 \mathrm{E}-08$ & 31,0 & 0,00 & $1,68 \mathrm{E}-09$ & 3,34E-09 & 0,1 & 0,00 \\
\hline & 3 & 301 & $2,09 \mathrm{E}-07$ & $5,21 \mathrm{E}-08$ & 36,5 & 0,00 & 1,18E-08 & $3,17 \mathrm{E}-08$ & 0,4 & 0,00 \\
\hline & 4 & 349 & $9,45 \mathrm{E}-06$ & $4,29 \mathrm{E}-08$ & 42,1 & 0,06 & $6,74 \mathrm{E}-06$ & $1,35 \mathrm{E}-05$ & 0,3 & 0,00 \\
\hline & 5 & 398 & $1,32 \mathrm{E}-06$ & 4,35E-08 & 47,8 & 0,03 & $9,15 \mathrm{E}-04$ & $1,61 \mathrm{E}-03$ & 0,4 & 0,03 \\
\hline & 6 & 454 & $6,03 \mathrm{E}-06$ & $4,07 \mathrm{E}-08$ & 54,1 & 0,10 & $6,40 \mathrm{E}-04$ & $1,28 \mathrm{E}-03$ & 0,5 & 0,03 \\
\hline & 7 & 506 & $4,61 \mathrm{E}-06$ & $3,65 \mathrm{E}-08$ & 59,6 & 0,09 & 1,00E-03 & $2,20 \mathrm{E}-04$ & 0,9 & 0,06 \\
\hline & 8 & 557 & $4,84 \mathrm{E}-06$ & $3,20 \mathrm{E}-08$ & 64,3 & 0,04 & 5,27E-04 & $1,15 \mathrm{E}-03$ & 0,7 & 0,02 \\
\hline & 9 & 614 & $1,15 \mathrm{E}-05$ & $3,23 \mathrm{E}-08$ & 70,4 & 0,27 & 3,28E-04 & $2,12 \mathrm{E}-05$ & 1,0 & 0,04 \\
\hline & 10 & 666 & $1,35 \mathrm{E}-05$ & $3,35 \mathrm{E}-08$ & 76,1 & 0,33 & $5,24 \mathrm{E}-04$ & 7,19E-04 & 1,1 & 0,08 \\
\hline
\end{tabular}


aumento pode ser explicado pela introdução de interrupções cíclicas no direito de passagem da corrente de tráfego principal que não existia antes da implantação. O percentual de veículos em conflito também aumentou com a implantação semafórica. Entretanto, esse comportamento fica mais aparente somente nos níveis mais altos de volume. Ainda nesse tipo de interação, o $\mathrm{CPI} /$ veic, a porcentagen de veículos interagindo e em conflito, cresceram com o aumento do volume, indicando que a redução do espaçamento médio existente elevou o risco de colisões traseiras. O CPI85, por outro lado, apresentou redução com o aumento do volume para os dois tipos de controle. Essa redução pode ter sido causada pelo fato do aumento de volume ter restringido os condutores mais agressivos de atingirem elevadas velocidades, tornando o fluxo geral mais homogêneo.

Para as interações transversais (possíveis colisões laterais), os resultados indicam uma menor frequência (porcentagem de veiculos interagindo) e maior severidade (CPI/veh) quando comparado às interações longitudinais. Contrariamente ao esperado, o $\mathrm{CPI} /$ veic não apresentou redução em todos os níveis de volume após a sinalização. Para os níveis mais altos de volume este indicador foi maior para a interseção semaforizada. Pelo fato de ter sido permitida a "esquerda forçada" na simulação, acredita-se que com o aumento do volume os veículos passaram a formar pelotões mais uniformes, tornando difíceis e mais arriscadas as conversões à esquerda.

Houve um decréscimo no percentual de veículos interagindo transversalmente com a introdução do semáforo em virtude da eliminação de quase todos os pontos de conflitos transversais. Nas interseções não semaforizadas, o percentual de veículos interagindo transversalmente decresce após um dado nível de flu- xo veicular, provavelmente pela impossibilidade de alguns veículos cruzar a interseção durante tempo total da simulação (15 minutos).

Uma análise de variância (ANOVA) dos valores do $\mathrm{CPI} /$ veic foi realizada para verificar a influência do volume e do tipo de controle nesse indicador, considerados apenas dois níveis de volume reunidos em dois grupos, a saber: 1) Fluxo reduzido (níveis 1, 2 e 3); e 2) Fluxo elevado (níveis 8,9 e 10). Os resultados da ANOVA para as interações longitudinais e transversais estão apresentados nas Tabelas 3 e 4, respectivamente.

Os resultados da Tabela 3 confirmam o volume, tipo de controle e a interação entre as duas variáveis como sendo estatísticamente significante. Comprovase, desta forma, a afirmação anterior de que o nível médio das interações veiculares longitudinais (CPI/veic) aumenta com o volume e com a intodução da sinalização semafórica.

De modo semelhante, pela Tabela 4, observa-se que todas as variáveis consideradas na ANOVA foram estatisticamente significantes. Confirma-se, portanto, a assertiva prévia de que, para as interações transversais, o CPI/veic aumenta com o volume independente do tipo de controle. Além disso, a introdução do semáforo reduziu as interações transversais para os níveis mais baixos de volume (até 1200vph), ocorrendo o inverso nos níveis mais altos testados (a partir de 2200vph).

\section{CONCLUSÕES}

Este artigo apresentou um modelo para a avaliação do desempenho da segurança viária utilizando a simulação microscópica. Em sua premissa básica, o modelo assume que a segurança viária se relaciona com o ní-

Tabela 3. Two-way ANOVA - Interações longitudinais (CPI/veic)

\begin{tabular}{lrrrrr}
\hline Fonte & $\begin{array}{c}\text { Soma dos } \\
\text { quadrados } \\
\text { Tipo III }\end{array}$ & $\begin{array}{c}\text { Graus de } \\
\text { liberdade }\end{array}$ & $\begin{array}{c}\text { Quadrados } \\
\text { médios }\end{array}$ & Valor-F & Significância. \\
\hline Volume & 15,74 & 1 & 15,74 & 44,20 & 0,00 \\
Controle & 7,03 & 1 & 7,03 & 19,74 & 18,81 \\
Volume * Controle & 6,70 & 1 & 6,70 & 0,00 \\
Erro & 62,66 & 176 & 0,36 & \\
Total & 108,47 & 180 & & \\
\hline
\end{tabular}

Tabela 4. Two-way ANOVA - Interações transversais (CPI/veic)

\begin{tabular}{lrrrrr}
\hline Fonte & $\begin{array}{c}\text { Soma dos } \\
\text { quadrados } \\
\text { Tipo III }\end{array}$ & $\begin{array}{c}\text { Graus de } \\
\text { liberdade }\end{array}$ & $\begin{array}{c}\text { Quadrados } \\
\text { médios }\end{array}$ & Valor-F & Significância. \\
\hline Volume & 21326,5 & 1 & 21326,50 & 5,95 & 0.02 \\
Controle & 18432,12 & 1 & 18432,12 & 5,15 & 0.02 \\
Volume * Controle & 15209,71 & 1 & 15209,71 & 4,25 \\
Erro & 630358,08 & 176 & 3581,58 & & \\
Total & 743035,21 & 180 & & \\
\hline
\end{tabular}


vel de turbulência observado no sistema de tráfego em função das interações longitudinais e transversais entre diferentes veículos ao longo do tempo.

$\mathrm{O}$ indicador proxy de segurança utilizado no modelo foi o índice de potencial para acidentes (CPI). O CPI é calculado a partir da taxa de desaceleração necessária para evitar a colisão e de um componente estocástico para a capacidade máxima de desaceleração de um veículo. Como plataforma de simulação utilizou-se o aplicativo $\operatorname{VISSIM}^{\odot} 4.3$ pela natureza de seus algoritmos, capacidade de customização de saída de dados e nível de detalhes representados.

O modelo foi ilustrado através de uma aplicação em interseções isoladas. Nesta aplicação investigou-se o desempenho da segurança viária de interseções controladas por placa de PARE e por semáforos. Os resultados indicam que a introdução do semáforo aumentou o percentual de veículos interagindo além do $\mathrm{CPI} /$ veic médio e CPI85 das interações longitudinais (possíveis colisões traseiras). Observou-se ainda o crescimento do percentual de veículos interagindo com o aumento do fluxo veicular.

O percentual de veículos interagindo transversalmente foi reduzido com a instalação do semáforo pela diminuição dos pontos de conflito, entretanto, a severidade das interações devido às conversões à esquerda aumentou consideravelmente, em virtude da formação de pelotões veiculares. A relação entre o CPI/veic, volume, tipo de controle de circulação e tipo de interação foi confirmado por uma análise de variância e testes de hipóteses efetuados com nível de significância de $5 \%$.

Os resultados apresentados nesta pesquisa indicam que a microssimulação tem um potencial considerável para ser utilizada em estudos de segurança viária. No intuito de consolidar esse tipo de metodologia em estudos de segurança viária recomendam-se esforços no sentido de desenvolver algoritmos de microssimulação mais abrangentes em relação ao comportamento humano como condutor, aperfeiçoamento dos processos de calibração e validação dos algoritmos existentes e o desenvolvimento de procedimentos de validação dos indicadores proxy obtidos utilizando indicadores de segurança viária mais objetivos.

\section{AGRADECIMENTOS}

Os autores agradecem ao Professor Felipe Loureiro por seus comentários e sugestões que permitiram aprimorar esse artigo e a CAPES pela bolsa de doutorado pleno no exterior concedida ao primeiro autor, culminando com a pesquisa aqui apresentada.

\section{REFERÊNCIAS BIBLIOGRÁFICAS}

Archer, J. (2005) Methods for the assessment and prediction of traffic safety at urban intersection and their application in microsimulation modelling. Tese de Doutorado, Department of Infrastructure. Royal Institute of Technology, Sweden.
Barceló, J.; A. Dumont; L. Montero; J. Perarnau e A. Torday (2003) Safety indicators for microsimulation-based assessments. In Proceedings of the $82^{\text {nd }}$ Annual Meeting of the Transportation Research Board, Washington, D.C., USA.

Cooper, D.F. e N. Ferguson (1976) A conflict simulation model. Traffic Engineering and Control. London. UK, p. 306-309.

Cunto, F.J.C. (2008) Assessing Safety Performance of Transportation Systems using Microscopic Simulation Tese de Doutorado, Department of Civil and Environmental Engineering, University of Waterloo, Ontário, Canadá.

Cunto, F.J.C. e C.F.G. Loureiro (2009) O Uso da Microssimulação na Avaliação do Desempenho da Segurança Viária. Artigo Submetido ao XXIII Congresso de Pesquisa e Ensino em Transportes, ANPET, Vitória, ES.

Darzentas, J.; D.F. Cooper; P.A. Storr e M.R. McDowell (1980). Simulation of road traffic conflicts at T-junctions. Simulation. p. 155164.

FHWA (2003) Manual on Uniform Traffic Control Devices - MUTCD. Federal Highway Administration. US Department of Transportation, Washington, D.C., USA

Gettman, D. e L. Head (2003) Surrogate safety measures from traffic simulation models. Federal Highway Administration report RD-03050. McLean, VA, USA.

Hauer, E. (1978). Traffic conflict surveys: some study design considerations. Technical report, Transportation and Road Research Laboratory - TRRL, UK.

Hauer, E. (2002). Observational Before-after Studies in Road Safety. (1 ${ }^{\mathrm{a}}$ ed.). Pergamon.

Huguenin, F.; A. Torday e A. Dumont (2005) Evaluation of traffic safety using microsimulation. In Proceedings of the 5th Swiss Transport Research Conference - STRC, Ascona, Suíça.

Hydén, C. (1987) The development of a method for traffic safety evaluation: The swedish traffic conflicts technique. Bulletin 70. Department of Traffic Planning and Engineering, Lund University, Lund, Sweden.

Minderhoud, M. e P. Bovy (2001). Extended time to collision measures for road traffic safety assessment. Accident Analysis and Prevention, v.33, p. 89-97.

PTV AG (2007) VISSIM 4.3 User Manual. Planung Transport Verkehr AG. Alemanha.

Saunier, N. e T. Sayed (2007) Automated road safety analysis using video data. In Transportation Research Record: Journal of the Transportation Research Board, No. 2019, TRB, National Research Council, Washington, D.C., p. 57-64.

Wiedemann, R. (1974). Simulation of road traffic flow. Reports of the Institute for transport and communication. v. 8, University of Karlsruhe, Alemanha.

Wiedemann, R. e U. Reiter (1992) Microscopic traffic simulation: the simulation system MISSION, background and actual state, $C E C$ Project ICARUS (V1052) Final Report, v. 2, Appendix A. Brussels: CEC, Bruxelas, Bélgica.

Xin, W.; J. Houdos; P. Michalopoulos e G. Davis (2008) The less-thanperfect driver: a model of collision-inclusive car-following behavior. In Proceedings of the 87th Annual Meeting of the Transportation Research Board, Washington D.C., USA. 